ORIGINAL ARTICLE

\title{
Patellar taping does not change the amplitude of electromyographic activity of the vasti in a stair stepping task
}

\author{
S M Cowan, P W Hodges, K M Crossley, K L Bennell
}

Br J Sports Med 2006;40:30-34. doi: 10.1136/bjsm.2005.018499

See end of article for authors' affiliations

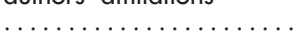

Correspondence to: Dr Cowan, School of Physiotherapy, University of Melbourne, Melbourne, Victoria 3010, Australia: s.cowan@unimelb.edu.au

Accepted 14 June 2005

\begin{abstract}
Objectives: To investigate the effect of patellar taping on the amplitude of electromyographic activity (EMG) of vasti activation in subjects with and without patellofemoral pain (PFP).

Methods: Ten participants with PFP and 12 asymptomatic controls were recruited to the study. The study was designed as a randomised crossover trial. Participants completed a stair stepping task. Three experimental conditions were assessed: no tape, therapeutic medially directed tape, and placebo vertically directed tape. The main outcome measure was the EMG amplitude of the vastus medialis obliquus and vastus lateralis during the concentric phase of stair stepping.

Results: The application of medially directed therapeutic tape significantly decreased pain in subjects with PFP. However, application of tape over the patella (therapeutic or placebo) did not alter the amplitude of vasti EMG when either the PFP or control participants completed the concentric stair stepping task.

Conclusion: The results of this study indicate that the positive clinical effects of medially directed therapeutic tape are not due to changes in EMG amplitude of the vasti muscle. Thus other effects such as changes in timing of contraction of the vasti are more likely candidates for the mechanism of efficacy.
\end{abstract}

A pplication of medially directed tape over the patella was originally developed by McConnell ${ }^{1}$ as a novel component of treatment for patellofemoral pain (PFP). The original hypothesis was that patellar taping normalised patellar tracking by mechanically shifting the patella in a medial direction. ${ }^{1}$ Although the evidence is inconclusive as to whether patellar position is actually altered by the application of tape to the skin, ${ }^{2-4}$ immediate pain relief with patellar taping is well documented. ${ }^{24-9}$

Mechanisms to explain the apparent pain relieving effect of patellar tape are unclear. It is possible that patellar tape affects the neuromotor control of the vasti. Recent evidence shows that the application of patellar tape alters the relative timing of the vasti in people with PFP. ${ }^{11}{ }^{11}$ Another aspect of neuromotor control, which may be important, is the relative activation of the vasti. Although McConnell $^{1}$ reported preliminary evidence that vastus medialis obliquus (VMO) activity was enhanced with a manual medial glide of the patella during a maximal quadriceps contraction in two asymptomatic subjects, there has been conflicting evidence in the subsequent literature. Two studies have reported differences in the activation of VMO and vastus lateralis (VL) with patellar taping in subjects with and without PFP, ${ }^{12}{ }^{13}$ others have reported no effects. ${ }^{714}$ The inconsistent findings in the literature may be due to factors such as different tasks and differences in the definition of PFP and in the methods used to normalise the electromyographic (EMG) activity. EMG normalisation may be particularly important as this may influence the variability of the data. ${ }^{16-20}$ One method that may be particularly sensitive for detecting differences is to normalise EMG activity to a reference condition without tape. This method has not previously been reported in PFP studies. Previous studies that have normalised EMG amplitude to a maximal contraction in patients with PFP may have failed to find significance because of variability associated with performing a pain provoking activity.

The purpose of this study was to investigate the effect of patellar taping on the amplitude of VMO and VL EMG in patients with PFP and in asymptomatic controls.

\section{METHODS}

\section{Participants}

Ten participants diagnosed with PFP by an experienced musculoskeletal physiotherapist and 12 asymptomatic controls were recruited to the study. Control participants were recruited from the staff and student population at the School of Physiotherapy. Participants with PFP were recruited from sports medicine practices, physiotherapists, and sporting clubs. The inclusion and exclusion criteria were based on previous studies ${ }^{21} 22$ and have been reported in full elsewhere. ${ }^{10}$ The participants in this study were also participants in a previously reported study that describes EMG onset timing of the vasti during the stair stepping task. ${ }^{10}$

The study was approved by The University of Melbourne Human Research Ethics Committee. All participants provided written informed consent.

\section{Interventions}

Participants underwent three experimental interventions, applied to their dominant (controls) or most painful (PFP group) knee, randomised using a latin square design (therapeutic tape, placebo tape, no tape) within the one testing session. Subjects rested for five minutes between each intervention to ensure that any effect of taping had disappeared before the start of the next task. ${ }^{10}$

\section{Therapeutic taping protocol}

Therapeutic taping was applied in a standardised sequence until the participant's pain was reduced by at least $50 \%$ on a $10 \mathrm{~cm}$ visual analogue scale during a pain provoking activity. Therapeutic taping procedures included medial glide and tilt, anterior tilt, rotation, and fat pad unloading. ${ }^{10}$ Skin protection was provided using a non-rigid, hypoallergenic tape (Fixomull; Beiersdorf Australia, North Ryde, NSW, Australia), and rigid zinc oxide tape (Endura-tape; Victor Endura-Tape, Clareville, Australia) was used to tape the

Abbreviations: EMG, electromyographic; PFP, patellofemoral pain; VL, vastus lateralis; VMO, vastus medialis obliquus 
patella. All control participants were taped with standard therapeutic taping (medial glide and tilt).

\section{Placebo taping protocol}

A non-rigid hypoallergenic tape (Fixomull) was applied to the skin in a vertical direction over a flexed knee with the participant sitting. ${ }^{10}$ The tape was placed with care such that the alignment of the patella was not visibly altered and to ensure that the knee could be fully flexed without restriction.

\section{Pain measures}

After each intervention, participants completed a $10 \mathrm{~cm}$ visual analogue scale for average pain experienced during the stair stepping task.

\section{EMG recordings}

Surface electrodes were used to record the EMG activity of VMO and VL. Before electrode placement, electrical impedance was reduced to less than $5 \mathrm{k} \Omega$ by shaving, swabbing with alcohol, and gently abrading the skin. Silver/silver chloride electrodes (Graphics Control Corp, Medical Equipment Services, Richmond, Australia) were placed over the muscle bellies of VMO and VL with an interelectrode distance of $22 \mathrm{~mm}$. The VMO electrodes were placed over the muscle belly $40 \mathrm{~mm}$ superior to and $30 \mathrm{~mm}$ medial to the superomedial patella border and were oriented $55^{\circ}$ to the vertical. The VL electrodes were placed $100 \mathrm{~mm}$ superior and 60-80 $\mathrm{mm}$ lateral to the superior border of the patella and orientated at $15^{\circ}$ to the vertical. ${ }^{11}{ }^{23}$ The ground electrode was placed over the tibial tubercle.

\section{Movement analysis}

A PEAK movement analysis system (Peak Performance Technology Inc, Englewood, Colorado, USA) was used to identify the concentric phase of the stair stepping task and the time taken to complete the phase (heel strike to toe off). Movement data were sampled at $50 \mathrm{~Hz}$ using a single camera placed perpendicular to the centre of the lateral side of the stair apparatus.

\section{Stair apparatus}

The stairs were placed in the centre of a $5 \mathrm{~m}$ walkway. The dimensions of the stairs were based on a previous study ${ }^{24}$ and consisted of a $60 \mathrm{~cm}$ platform with two steps of $20 \mathrm{~cm}$ height on both sides.

\section{Procedure}

Participants completed a stair stepping task at a rate of 96 steps per minute paced by an external metronome. EMG activity of VMO and VL was recorded during the stance phase of the middle step during ascent and descent for five consecutive trials for each intervention. ${ }^{24}$

EMG data were pre-amplified $(10 \times)$, band pass filtered at 20-500 Hz, sampled at $1 \mathrm{kHz}$, and 12 bit A-D converted (Associative Measurement Pty, Ltd, North Ryde, NSW, Australia).

\section{EMG data analysis}

Root mean square EMG amplitude was calculated for 200 milliseconds after the onset of EMG which was identified and reported previously. ${ }^{10}$ The 200 millisecond window was chosen as it was found to be representative of the concentric contraction in most participants. EMG amplitude in the placebo tape and therapeutic tape conditions was expressed as the percentage change in activity from the amplitude recorded in the control condition without application of tape. This form of analysis indicates a change in the activity of the muscles due to the application of tape and removes the need to normalise the EMG to a maximal contraction. In addition, the EMG activity ratio of VMO to VL was calculated for no tape, placebo tape, and tape condition. The assessor of the EMG data was blinded to group allocation, taping condition, and muscle. EMG data were analysed for the concentric phase of the stair stepping task only.

\section{Statistical analysis}

All data were analysed using Statistical Package for Social Sciences (SPSS; Norusis/SPSS Inc, Chicago, Illinois, USA). The data were assessed for normality and homogeneity of variance.

\section{Relative magnitude of vasti activity}

A three way (group $\times$ taping condition $\times$ muscle) mixed measures analysis of variance was used to compare the percentage change of taping conditions (placebo and therapeutic) between muscles (VMO and VL) and groups (PFP and control). The $\alpha$ level was set at $\mathrm{p}<0.05$.

\section{EMG activity ratio}

The EMG activity ratio of VMO to VL was calculated (EMG activity ratio $=\mathrm{VMO} / \mathrm{VL}$ ). Separate repeated measures analysis of variance were used to compare the effect of taping (no tape, control tape, therapeutic tape) in the two groups (PFP and control). The $\alpha$ level was set at $\mathrm{p}<0.05$.

\section{Pain measures}

Separate repeated measures analysis of variance were used to compare average pain levels for the three taping conditions in the PFP group and to compare the time taken to complete the stair stepping task between conditions. Post hoc paired $t$ tests were used to determine specific differences.

\section{Correlations}

Univariate correlations were sought between the percentage change in vasti activity, pain, change in onset timing (using the previously published data set), baseline onset timing, age, height, weight, and sex.

\section{RESULTS}

The basic data (mean (SD)) for the participants with PFP were: age 23.0 (8.0) years; height $1.67(0.10) \mathrm{m}$; weight 59.3 (10.1) kg; body mass index $21.10(1.82) \mathrm{kg} / \mathrm{m}^{2}$. The same data for the control participants were: 19.5 (1.4) years, 1.71 (0.11) m, $60.8(8.1) \mathrm{kg}$ and $20.75(1.39) \mathrm{kg} / \mathrm{m}^{2}$. Independent $t$ tests revealed no significant differences between the two groups for these variables.

\section{Adverse reactions}

No participant reported adverse symptoms while wearing either form of tape, nor did the investigator observe any adverse skin responses.

\section{Pain measures}

When participants with PFP completed the stair stepping task, they had a mean (SE) pain of 3.5 (0.5). In the PFP group, therapeutic taping of the patellofemoral joint resulted in changes in the mean pain reported by the participants as they completed the stair stepping task $(p<0.0001)$. Pain was significantly less in the therapeutic taped condition than in both the placebo $(\mathrm{p}<0.0001)$ and no tape condition $(p<0.0001)$. There was no difference between the no tape and placebo taped condition $(\mathrm{p}=0.591)$. There were no differences in the time taken to complete the stair stepping task between taping conditions in either group $(\mathrm{p}=0.634)$.

\section{Relative magnitude of vasti activation}

Table 1 summarises the changes in EMG activity for the PFP and control participants with therapeutic taping. Figure 1 
Table 1 Number of participants who displayed an increase or decrease in electromyographic (EMG) activity of vastus medialis obliquus and vastus lateralis muscles and mean (SD) percentage change in EMG magnitude

\begin{tabular}{|c|c|c|c|c|c|c|c|c|}
\hline & \multicolumn{4}{|c|}{ Vastus medialis obliquus } & \multicolumn{4}{|c|}{ Vastus lateralis } \\
\hline & Increase & Percentage change & Decrease & Percentage change & Increase & Percentage change & Decrease & Percentage change \\
\hline PFP & 7 & $34(22)$ & 3 & $23(15)$ & 4 & $22(31)$ & 6 & $6(7)$ \\
\hline Control & 6 & $8(25)$ & 6 & $23(13)$ & 4 & $4(3)$ & 8 & $17(10)$ \\
\hline
\end{tabular}

shows the percentage change in vasti activity that occurred with therapeutic and placebo tape in the PFP and control participants. The mixed analysis of variance revealed that there were no differences between the control and PFP group $(\mathrm{p}=0.215)$, there were no differences between muscles $(\mathrm{p}$ $=0.136)$ or taping conditions $(\mathrm{p}=0.784)$, and there were no significant interactions between muscle and group $(\mathrm{p}=$ $0.472)$, tape and group $(\mathrm{p}=0.115)$, muscle and tape $(\mathrm{p}=$ $0.232)$, or muscle, tape, and group $(\mathrm{p}=0.227)$.

\section{EMG activity ratio}

Figure 2 shows the EMG activity ratio (VMO/VL) in the three taping conditions for the control and PFP groups. The repeated measures analysis of variance revealed no differences between taping conditions in either the control ( $\mathrm{p}=$ $0.171)$ or the PFP group $(p=0.256)$.

\section{Correlations}

No single factor or combination of factors were found to predict change in the amplitude of EMG activation of the vasti with the application of therapeutic or placebo tape. There were no significant correlations between the change in vasti activity levels and EMG baseline and change in onset timing differences, pain or age, height, weight, and sex.

\section{DISCUSSION}

This study found that taping had no consistent effect on the relative magnitude of activation of the vasti in participants with PFP, despite the fact that tape altered both pain and EMG onset timing in the same population. ${ }^{10}$ Thus the findings do not support the hypothesis that changes in the relative activation of the vasti with therapeutic patellar tape

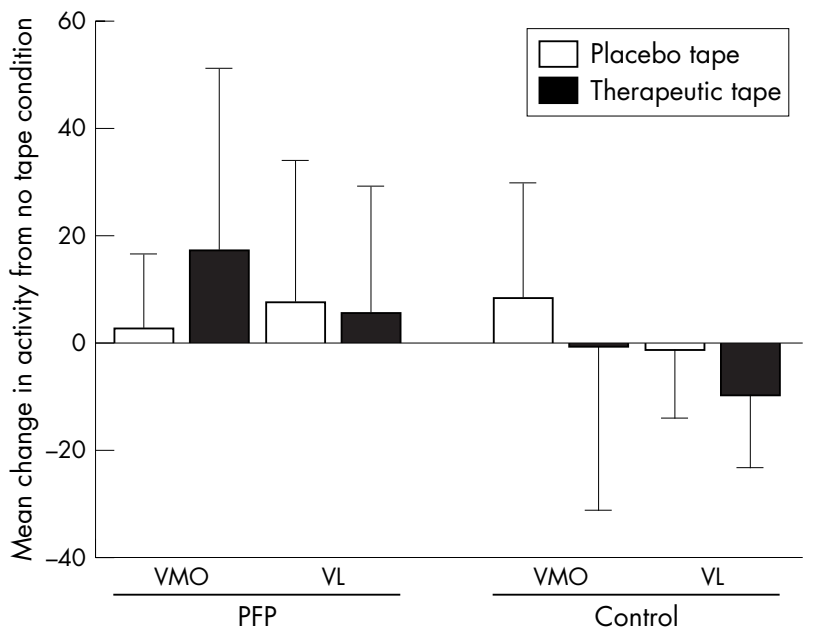

Figure 1 Mean (SD) change in vasti activity with placebo and therapeutic taping conditions compared with the no tape condition in the control participants and those with patellofemoral pain (PFP). VL, vastus lateralis; VMO, vastus medialis obliquus. There were no significant differences.

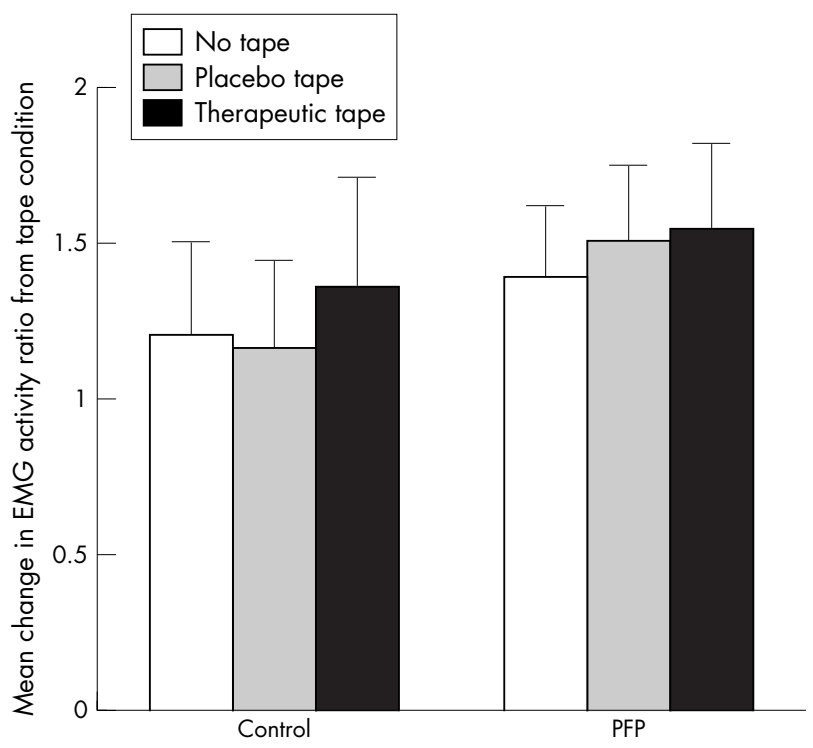

Figure 2 Mean (SE) electromyographic (EMG) activity ratio of vastus medialis obliquus versus vastus lateralis in the control and patellofemoral pain (PFP) groups. There were no significant differences.

may be responsible for the well documented pain relief associated with patellar taping.

The finding that therapeutic taping did not affect the magnitude of vasti activation in subjects with PFP agrees with the findings of a number of previous studies. ${ }^{7}{ }^{14} 15$ There are a number of possible explanations for the lack of change in EMG activation levels with patellar tape. Firstly, patellar tape may improve the lever arm of the quadriceps through a change in knee flexion angle. A change in knee flexion angle with patellar taping ${ }^{9}$ may have placed the vasti in a more optimal position for force generation. This improved length tension relation may have been reflected by a change in EMG onset timing, and, as a result, more quadriceps force could have been generated at the same muscle activation level. This may have resulted in the change of EMG onset timing (or vice versa), which may contribute to an increased knee extensor moment without an increase in quadriceps force. Although quadriceps force was not measured in the present study, knee extensor moment has been found to improve with patellar tape. ${ }^{8526}$ Secondly, the application of therapeutic tape may have resulted in a different lower limb muscle strategy to accomplish the task, which may be reflected in the change in EMG onset timing of the vasti without a change in the level of vasti activation. This could be further investigated with the assessment of other lower limb muscles, such as the vastus intermedius, rectus femoris gluteals, hamstrings, and calf muscles, and the assessment of three dimensional kinematics all of which may have contributed to a change in strategy and a change in timing with the application of tape. 
The findings contrast with a number of studies that have found that patellar tape affects the magnitude of activation of the vasti in PFP..$^{12}$ However, one of these papers are presented only in abstract form, making detailed critique of their methods difficult. $\mathrm{Ng}$ et al $^{13}$ used a ratio of VMO to VL and found patellar taping actually decreased the activation of VMO, which is in contrast with the proposed effect of patellar tape to increase VMO activity. The authors explain that this confounding result may be related to the mechanical effect of tape but that further investigation is required. Also worthy of consideration is that all participants may not respond in the same way to application of therapeutic tape, and thus a group effect may not be found unless responders and nonresponders can be identified a priori. Indeed seven of the 10 participants with PFP responded positively to the application of therapeutic tape-that is, increased the amplitude of VMO-with a mean increase in this group of 34\%. However, statistical analysis did not identify predictive factors for those who responded positively.

The finding that patellar tape did not affect the magnitude of activation of the vasti in control participants concurs with our previous research, which found that patellar taping does not affect EMG onset timing in asymptomatic people. ${ }^{10}$ This may be because they generally already have an optimal strategy, and, to have an effect on the magnitude of activation, a deficit must exist to begin with.

The vertical untensioned taping procedure was included to control for a placebo effect. The study shows that placebo tape did not affect vasti activity, which is in agreement with previous studies that have found that placebo taping does not change the level of activation of $\mathrm{VL}^{27}$ pain, ${ }^{102125}$ or EMG onset timing. ${ }^{10}$ However, a limitation with the placebo taping procedure used in this study relates to the fact that the tape was not placed over the same area of skin as the therapeutic tape.

\section{Limitations}

It is possible that a true difference in EMG activity between taping conditions does exist but was not detected with our small sample size and relatively large variability (the observed power at $\alpha=0.05$ was 0.11 ). However, a post hoc power analysis $(\alpha=0.05$ power $=0.8)$ revealed that a sample size of greater than 175 subjects would be required for the observed difference of $16 \%$ seen with the application of therapeutic tape to be significant. Even with a significant difference, the clinical relevance of such a small difference in EMG activity is perhaps questionable.

Another possible cause of the failure to find a difference in EMG activity levels with therapeutic taping relates to the method of magnitude determination. It is possible that the technique used was insensitive to detect the size of change observed in this study. The technique uses the untaped condition as a reference point for "normal" EMG activity. As the electrodes were not removed between taping conditions, any effect of therapeutic tape or placebo tape would be seen as a change in the activity of VMO or VL relative to the untaped condition. Thus the change can be expressed as a

\section{What is already known on this topic}

- Controversy exists as to whether patellar tape can alter the magnitude of activation of the vasti in people with patellofemoral pain

- One possible explanation for the inconsistent findings in the literature may be the different techniques used to normalise electromyographic activity of the vasti

\section{What this study adds}

- This study provides further evidence that patellar tape does not change vasti activity in people with patellofemoral pain

- A within subject design allows electromyographic data to be normalised without the use of painful maximal contraction, which can add to data variability

percentage change. This technique has previously only been used in asymptomatic populations, ${ }^{28}{ }^{29}$ but theoretically should be very sensitive to change. To explore the possibility that a relative change in the magnitude of vasti activation occurred, the amplitude of VMO was compared directly with that of VL. However, this also failed to show any significant changes. Another possibility is that the application of tape may have stretched the skin, moving it with respect to the muscle so that a different area of the muscle was being recorded after the application of tape. Lastly, the possibility of carry over effects from the different taping conditions cannot be conclusively ruled out because of the small sample size and latin square design.

\section{CONCLUSION}

The results of this study provide further insight into the effect of patellar taping on neuromotor control of the vasti. The finding that patellar taping had no effect on the levels of magnitude of vasti activation in both painful and non-painful subjects supports most previous research and indicates that the positive clinical effects of medially directed therapeutic tape are not due to changes in EMG amplitude of the vasti muscle. Thus other effects such as changes in timing of contraction of the vasti are more likely candidates for the mechanism of efficacy.

\section{ACKNOWLEDGEMENTS}

This study was supported by a grant from the Physiotherapy Research Foundation. SC, KC, and $\mathrm{PH}$ are funded by the National Health and Medical Research Council.

\section{Authors' affiliations}

S M Cowan, K M Crossley, K L Bennell, School of Physiotherapy, University of Melbourne, Melbourne, Victoria, Australia P W Hodges, University of Queensland, Brisbane, Australia

Competing interests: none declared

\section{REFERENCES}

1 McConnell J. The management of chondromalacia patellae: a long term solution. Aust J Physiother 1986:32:215-23.

2 Bockrath K, Wooden C, Worrell T, et al. Effects of patella taping on patella position and perceived pain. Med Sci Sports Exerc 1993;25:989-92.

3 Roberts JM. The effect of taping on patellofemoral alignment: a radiological study. In: MPAA Adelaide: 6th Biennial Conference 1989:146-51.

4 Somes S, Worrell TW, Corey B, C. D. I. Effects of patellar taping on patellar position in the open and closed kinetic chain: a preliminary study. Journal of Sport Rehabilitation 1997;6:299-308

5 Kenna M. The effect of patellofemoral joint taping on pain during activity. In: MPAA Blue Mountains, Australia: 7th Biennial Conference 1991:134-9.

6 Herrington L, Payton CJ. Effects of corrective taping of the patella on patients with patellofemoral pain. Physiotherapy 1997;83:566-72.

7 Cerny K. Vastus medialis oblique/vastus lateralis muscle activity ratios for selected exercises in persons with and without patellofemoral pain syndrome. Phys Ther 1995;75:672-82.

8 Conway A, Malone T, Conway P. Patella alignment/tracking alteration: effect on force output and perceived pain. Journal of Isokinetic Exercise Science 1992;2:9-17.

9 Powers CM, Landel R, Sosnick T, et al. The effects of patellar taping on stride characteristics and joint motion in subjects with patellofemoral pain. J Orthop Sports Phys Ther 1997;26:286-91. 
10 Cowan SM, Bennell KL, Hodges PW. Therapeutic patellar taping changes the timing of vastii muscle activation in people with patellofemoral pain syndrome. Clin J Sport Med 2002;12:339-47.

11 Gilleard W, McConnell J, Parsons D. The effect of patellar taping on the onset of vastus medialis obliquus and vastus lateralis muscle activity in persons with patellofemoral pain. Phys Ther 1998;78:25-31.

12 Christou EA, Carlton LG. The effect of knee taping on the EMG activity of the vastus medialis oblique and vastus lateralis muscles. Med Sci Sport Exerc 1997:29:S211.

$13 \mathrm{Ng}$ GY, Cheng JM. The effects of patellar taping on pain and neuromuscular performance in subjects with patellofemoral pain syndrome. Clin Rehabil 2002; 16:821-7.

14 Millar AL, Berglund K, Blake B, et al. Effects of patellofemoral taping on knee pain and EMG activity of the quadriceps. In: 12th International Confederation of Physical Therapy. Washington, DC, 1999.

15 Salsich GB, Brechter JH, Farwell D, et al. The effects of patellar taping on knee kinetics, kinematics, and vastus lateralis muscle activity during stair ambulation in individuals with patellofemoral pain. J Orthop Sports Phys Ther 2002;32:3-10.

16 Knutson LM, Soderberg GL, Ballantyne BT, et al. A study of various normalisation procedures for within day electromyographic data. J Electromyogr Kinesiol 1994;4:47-59.

17 Winter DA. Electromyogram recording, processing, and normalisation procedures and considerations. Journal of Human Muscle Performance 1991; 1:5-15.

18 Yang JF, Winter DA. Electromyography reliability in maximal and submaximal isometric contractions. Arch Phys Med Rehabil 1983;64:417-20.

19 Yang JF, Winter DA. Electromyographic amplitude normalisation methods: improving their sensitivity as diagnostic tools in gait analysis. Arch Phys Med Rehabil 1984;65:517-21.
20 Burden A, Bartlett R. Normalisation of EMG amplitude: an evaluation and comparison of old and new methods. Med Eng Physics 1999;21:247-57

21 Crossley K, Bennell K, Green S, et al. Physical therapy for patellofemoral pain: a randomised, double-blind, placebo controlled trial. Am J Sports Med 2002;30:857-65.

22 Harrison EL, Sheppard MS, McQuarrie AM. A randomised controlled trial of physical therapy treatment programs in patellofemoral pain syndrome. Physiother Canada 1999:93-100.

23 Basmajian J, Blumenstein R. Electrode placement in EMG biofeedback. Baltimore: Williams and Wilkins, 1980.

24 Cowan SM, Bennell KL, Hodges PW. The test retest reliability of the onset of concentric and eccentric vastus medialis obliquus and vastus lateralis electromyographic activity in a stair stepping task. Physical Therapy in Sport 2000; 1:129-36.

25 Handfield T, Kramer J. Effect of McConnell taping on perceived pain and knee extensor torques during isokinetic exercise performed by patients with patellofemoral pain. Physiother Canada, 2000;39-44.

26 Ernst GP, Kawaguchi J, Saliba E. Effect of patellar taping on knee kinetics of patients with patellofemoral pain syndrome. J Orthop Sport Phys Ther 1999;29:661-7.

27 Tobin S, Robinson G. The effect of McConnells vastus lateralis inhibition taping technique on vastus lateralis and vastus medialis activity. Physiotherapy 2000:86:173-83.

28 Hodges $\mathbf{P}$, Richardson CA. The influence of isometric hip adduction on quadriceps femoris activity. Scand J Rehabil Med 1993;25:57-62.

29 Kay D, Marino F, Cannon J, et al. Evidence for neuromuscular fatigue during high-intensity cycling in warm, humid conditions. Eur J Appl Physiol $2001 ; 84: 115-21$. 